\title{
Ottimizzazione delle misure strumentali con sistemi IOMS per il monitoraggio delle emissioni odorigene in impianti complessi
}

\author{
Raffaele Massimo ${ }^{1}$ \\ ${ }^{1}$ Affiliation not available
}

\begin{abstract}
Le emissioni odorigene generate da impianti industriali e di tutela ambientale sono spesso causa di potenziali fenomeni di molestia olfattiva in grado di generare malcontento nei cittadini che vi risiedono in prossimità e, per tale ragione, vanno monitorate per contenerle entro determinate soglie tramite azioni di controllo e mitigazione. La caratterizzazione quantitativa e qualitativa dei composti di tipo odorigeno risulta, tuttavia, ancora di complessa esecuzione, sia a causa della soggettività intrinseca della percezione olfattiva sia per effetto dell'influenza delle condizioni meteoclimatiche sui fenomeni di dispersione odorigena e, dunque, sui livelli di fastidio percepiti.

Fra le tecniche di misura generalmente impiegate per il monitoraggio degli odori, quelle senso-strumentali con applicazione dei nasi elettronici, più recentemente definiti IOMS (Instrumental Odour Monitoring Systems), rappresentano attualmente la soluzione più efficace per consentire l'investigazione della classe odorigena e dell'intensità di odore e sono in grado di monitorare gli odori in maniera continua ed in tempo reale, coniugando i vantaggi delle convenzionali tecniche di misura strumentali e sensoriali. Gli IOMS rappresentano la tecnologia con il più alto potenziale di sviluppo futuro e quindi risultano essere dei sistemi ulteriormente migliorabili. La mancanza di riferimenti tecnico-normativi in materia di emissioni odorigene rappresenta ancora un fattore limitante per l'impiego di tali dispositivi. Il quadro normativo in materia di odori, tuttavia, è in evoluzione, con l'emanazione di norme e regolamenti in materia sia in ambito nazionale che comunitario ed internazionale. La Norma UNI 11761:2019, in fase di revisione, definisce i requisiti tecnici e di gestione degli IOMS per la misurazione degli odori in aria ambiente, alle emissioni e indoor.
\end{abstract}




\section{I sistemi IOMS}

Le emissioni odorigene prodotte da impianti industriali e di tutela ambientale possono dar luogo a fenomeni di molestia olfattiva in grado di arrecare fastidi alla popolazione esposta e rappresentano una delle principali cause di reclami da parte degli operatori degli impianti e della popolazione $\left({ }^{1} ;^{2} ;{ }^{3} ;{ }^{4}\right)$, in parte allietati da operazioni di recupero e riutilizzo di materiali di scarto dei suddetti impianti $\left({ }^{5} ;{ }^{6} ;{ }^{7}\right)$, di utilizzo di fonti rinnovabili $\left({ }^{8}\right)$ e di trattamento delle acque con processi innovativi $\left({ }^{9} ;{ }^{10} ;{ }^{11} ;{ }^{12} ;{ }^{13} ;{ }^{14} ;{ }^{15} ;{ }^{16} ;{ }^{17}\right)$. L'esposizione prolungata a miscele odorigene gassose, pur non identificando una causa diretta di malattie, può provocare gravi danni alla salute come nausea, mal di testa e problemi respiratori $\left({ }^{18} ;{ }^{1} ;{ }^{19} ;{ }^{20} ;{ }^{21} ;{ }^{22} ;{ }^{23} ;{ }^{24} ;{ }^{25}\right)$. Per misurare e quantificare gli odori, attualmente è solito ricorrere a tre tipologie di tecniche: strumentali, sensoriali e senso-strumentali $\left({ }^{18} ;{ }^{26}\right)$. Le tecniche strumentali consentono di identificare e quantificare e i composti chimici caratteristici delle emissioni maleodoranti $\left({ }^{27} ;{ }^{28}\right)$, fornendo informazioni utili per il monitoraggio delle sorgenti di odore, ma richiedono tempi di applicazione abbastanza prolungati e costi di esecuzione esosi; invece, nell' applicazione delle tecniche sensoriali, come l'olfattometria dinamica, il naso umano funge da rilevatore degli odori non garantendo però un'elevata precisione a causa della natura soggettiva della percezione della sensazione in fase di valutazione $\left({ }^{18} ;{ }^{27} ;{ }^{2} ;{ }^{2} ;{ }^{26} ;{ }^{28}\right)$. Infine, le tecniche senso-strumentali identificano ad oggi una valida soluzione per attribuire alle emissioni la classe odorigena ed un valore rappresentativo dell'intensità di odore $\left({ }^{29}\right)$. Tra le tecniche senso-strumentali, i nasi elettronici, o Instrumental Odour Monitoring Systems (IOMS), consentono di effettuare il monitoraggio degli odori in continuo, sfruttando quindi i punti di forza sia delle tecniche di misura strumentali che di quelle sensoriali $\left({ }^{24}\right)$; al contempo, essi necessitano ancora di approfondite ricerche per identificare soluzioni nella stabilizzazione della temperatura di esercizio o per la relazione con i parametri operativi (temperatura, portata in ingresso nella camera di misura, ecc.) da parte delle resistenze dei sensori. 
Gli IOMS sono in grado di identificare gli odori utilizzando un array di sensori aspecifici e un insieme di algoritmi $\left({ }^{30} ;{ }^{19} ;{ }^{31} ;{ }^{32}\right)$; entrambi gli elementi sono stati interessati da costanti miglioramenti connessi al continuo perfezionamento delle tecnologie di apprendimento automatico $\left({ }^{33} ;{ }^{34} ;{ }^{35}\right)$. Il naso elettronico ha trovato ampia applicazione in diversi settori come agricoltura $\left({ }^{36} ;{ }^{37}\right)$, diagnosi medica $\left({ }^{38} ;{ }^{39}\right)$ monitoraggio ambientale $\left({ }^{40} ;{ }^{41}\right)$ e protezione della sicurezza alimentare $\left({ }^{42} ;{ }^{43}\right)$. Per determinate applicazioni, la progettazione della componente hardware del dispositivo e la selezione dei sensori più adeguati, oltre che dei metodi di estrazione e classificazione delle caratteristiche, rappresentano degli elementi fondamentali per rendere più performanti le prestazioni del naso elettronico.

\section{Ottimizzazione delle modalità operative degli IOMS}

L'ottimizzazione dei risultati delle modalità operative di addestramento, validazione, misura e gestione di un sistema IOMS non può prescindere da un'accurata analisi dei suoi parametri di performance, da una corretta gestione delle variabili operative e da un'oculata scelta delle tecniche statistiche di analisi ed elaborazione dei dati in input $\left({ }^{24}\right)$.

I parametri di performance di un sistema IOMS sono indicativi della sua capacità di realizzare efficaci e significativi modelli predittivi, la cui bontà viene verificata attraverso un confronto tra la classe di odore ed il valore di concentrazione attribuiti ai campioni dal dispositivo ed i corrispondenti elementi già noti in fase di acquisizione dei campioni da parte dello strumento. Tali parametri di performance sono forniti dallo IOMS in termini di accuratezza nell'attribuzione della classe odorigena e della concentrazione ai relativi campioni, errore, indici di precisione e di richiamo $\left({ }^{44}\right)$ e possono essere stimati sfruttando le differenti modalità di preprocessamento e le varie tecniche di elaborazione statistica dei dati implementate dai sistemi IOMS. Su queste stesse variabili si può ulteriormente intervenire o per poterne migliorare l'accuratezza o, qualora i risultati in output dallo strumento non siano ritenuti soddisfacenti, andando a controllare e a stabilizzare i parametri ope- 
rativi del dispositivo, fra cui sono degni di nota certamente la temperatura di acquisizione dello IOMS e i valori della portata in ingresso nella camera di misura dello strumento. Infatti, oscillazioni troppo elevate dei suddetti parametri operativi possono condizionare notevolmente i valori di resistenza acquisiti dai sensori e, di conseguenza, i risultati delle attività di monitoraggio. Generalmente, la legge che lega la temperatura di acquisizione dello IOMS ed i valori di resistenza dei sensori è correlata ad una relazione di inversa proporzionalità, variabile in funzione della tipologia di sensore, della modalità operativa con cui si sta lavorando ed anche dalla tipologia di campioni acquisiti dallo strumento e con cui si intendono costruire i modelli predittivi.

A tal proposito, per la loro realizzazione, è indispensabile scegliere la corretta tecnica di elaborazione statistica dei dati, la quale consente, servendosi a sua volta di una serie di algoritmi, di selezionare le informazioni provenienti dai diversi sensori, di assimilare le differenti risposte ed istruire il naso elettronico. I metodi di analisi dei dati si classificano generalmente in metodi non supervisionati e metodi supervisionati $\left({ }^{45}\right)$. La loro appropriatezza alle problematiche oggetto di studio dipende dalla loro complessità, dal numero di oggetti e dalle loro variabili e dalla capacità di calcolo del software. I metodi non supervisionati vengono solitamente impiegati per ottenere informazioni preliminari, per il riconoscimento iniziale delle dipendenze fra le uscite dei sensori (variabili indipendenti) e la classe di odore e/o la concentrazione (variabili dipendenti) oppure per definire eventuali valori anomali. Il loro vantaggio consiste nel fatto, che a causa della mancanza di una vera e propria fase di addestramento dello IOMS, basta una quantità irrisoria di dati in input per ottenere informazioni significative. Allo stesso tempo, i metodi non supervisionati come l'Analisi delle Componenti Principali (PCA) o l'Analisi dei Cluster (CA) non possono essere impiegati negli algoritmi di classificazione automatica, tranne che nelle operazioni di preelaborazione.

Al contrario, nei metodi supervisionati vengono forniti sia l'input che i risultati da ottenere per costruire il modello; dopo la fase di addestramento del sistema IOMS, gli input sono assegnati alle classi. Queste tecniche sono utilizzate di solito per la classificazione e la discriminazione tra 
campioni quando è presente un campione di riferimento con caratteristiche desiderabili, che è poi confrontato con gli altri campioni. I metodi supervisionati più comuni sono l'Analisi Lineare Discriminante (LDA), le Macchine a Vettori di Supporto (SVM) e le Reti Neurali Artificiali (ANN), a cui sono associate altre tecniche utilizzate meno frequentemente.

La corretta implementazione e le operazioni di gestione di questa serie di variabili consentono di ottimizzare al meglio tutte le modalità operative implementate dai sistemi IOMS e di sfruttarne al meglio le loro enormi potenzialità.

\section{Conclusioni}

Le emissioni odorigene dalle diverse tipologie di impianti costituiscono una problematica di assoluta importanza, poiché notevolmente incidenti sia sugli interventi di costruzione di nuovi impianti o di adattamento degli esistenti sia sul benessere della popolazione residente, specialmente in prossimità degli stessi. Fra le tecniche di monitoraggio e controllo degli odori, assumono sempre maggiore importanza le misure senso-strumentali, tra cui gli IOMS, ritenuti già allo stato attuale dei dispositivi efficienti, ma allo stesso tempo sottoposti a processi di continuo miglioramento per ottenere risultati sempre più soddisfacenti.

Le svariate applicazioni su scala globale di diversi prototipi di IOMS per le operazioni di misura e di controllo degli odori in diversi settori hanno dimostrato che tali dispositivi rappresentano una soluzione efficace ed accurata per il monitoraggio in continuo delle emissioni odorigene provenienti da impianti industriali complessi. Tuttavia, il controllo delle condizioni operative relative al funzionamento di questi dispositivi in tutte le loro modalità, oltre che la selezione delle più appropriate modalità di estrazione ed elaborazione dei dati, rappresentano degli aspetti fondamentali per garantire adeguati standard di performance dei sistemi IOMS e costituiscono gli obiettivi principali delle attività di ricerca attuali e future. 


\section{References}

1.Odour monitoring of small wastewater treatment plant located in sensitive environment. Water Science and Technology 58, (2008).

2.Odour Impact Assessment in Industrial Areas. Chemical Engineering Transactions 30, (2012).

3.A novel tool for odor emission assessment in wastewater treatment plant. Desalination and Water Treatment 55, (2015).

4.Odour control strategies for a sustainable nuisances action plan.. Global Nest Journal 18, (2016).

5.Environmental Technologies for the Sustainable Development of the Water and Energy Sectors. (IWA Publishing, 2020). doi:10.2166/9781789062328.

6.Naddeo, V. \& Korshin, G. Water energy and waste: The great European deal for the environment. Science of The Total Environment 764, 142911 (2021).

7.Naddeo, V. \& Taherzadeh, M. J. Biomass valorization and bioenergy in the blue circular economy. Biomass and Bioenergy 149, 106069 (2021).

8.Scannapieco, D., Naddeo, V. \& Belgiorno, V. Sustainable power plants: A support tool for the analysis of alternatives. Land Use Policy 36, 478-484 (2014).

9.Degradation of Gaseous VOCs by Ultrasonication: Effect of Water Recirculation and Ozone Addition. Frontiers in Water-Energy-Nexus-Nature-Based Solutions, Advanced Technologies and Best Practices for Environmental Sustainability (2019).

10.Oliva, G. et al.. Comparative evaluation of a biotrickling filter and a tubular photobioreactor for the continuous abatement of toluene. Journal of Hazardous Materials 380, 120860 (2019). 
11.Abdallah, H. et al.. Amino-functionalized mesoporous nano-silica/polyvinylidene fluoride composite as efficient ultrafiltration membrane. DESALINATION AND WATER TREATMENT 205, $63-75$ (2020).

12.Ali, A. A. A., Naddeo, V., Hasan, S. W. \& Yousef, A. F. Correlation between bacterial community structure and performance efficiency of a full-scale wastewater treatment plant. Journal of Water Process Engineering 37, 101472 (2020).

13.Water-Energy Nexus: Evaluation of the Environmental Impact on the National and International Scenarios. Frontiers in Water-Energy-Nexus-Nature-Based Solutions, Advanced Technologies and Best Practices for Environmental Sustainability (2020).

14.Naddeo, V. Development of environmental biotechnology and control of emerging biological contaminants: the grand challenge for a sustainable future. Water Environment Research 92 , 1246-1248 (2020).

15.Pervez, M. N. et al. A critical review on nanomaterials membrane bioreactor (NMs-MBR) for wastewater treatment. npj Clean Water 3, (2020).

16.Abdulkarem, E. et al.. Polyvinylidene fluoride (PVDF)--zirconium phosphate (-ZrP) nanoparticles based mixed matrix membranes for removal of heavy metal ions. Chemosphere 267, 128896 (2021).

17.Millanar-Marfa, J. M. J. et al.. Self-forming Dynamic Membranes for Wastewater Treatment. Separation \& Purification Reviews 1-17 (2021) doi:10.1080/15422119.2021.1887223.

18.Gostelow, P., Parsons, S. A. \& Stuetz, R. M. Odour measurements for sewage treatment works. Water Research 35, 579-597 (2001).

19.Performance Study of E-Nose Measurement Chamber for Environmental Odour Monitoring. Chemical Engineering Transactions 30, (2012). 
20.Belgiorno, V., Naddeo, V., Scannapieco, D., Zarra, T. \& Ricco, D. Ecological status of rivers in preserved areas: Effects of meteorological parameters. Ecological Engineering 53, 173-182 (2013).

21.Naddeo, V., Belgiorno, V., Zarra, T. \& Scannapieco, D. Dynamic and embedded evaluation procedure for strategic environmental assessment. Land Use Policy 31, 605-612 (2013).

22.Comparative evaluation of a biotrickling filter and a tubular photobioreactor for the continuous abatement of toluene. Journal of Hazardous Materials 380, (2019).

23. Volatile organic compounds (VOCs) control by combining bio-scrubber and ozone pretreatment. Global NEST: the international Journal (2020) doi:10.30955/gnj.003298.

24.Oliva, G. et al.. Next-generation of instrumental odour monitoring system (IOMS) for the gaseous emissions control in complex industrial plants. Chemosphere 271, 129768 (2021).

25.Senatore, V. et al.. Innovative membrane photobioreactor for sustainable $\mathrm{CO} 2$ capture and utilization. Chemosphere 273, 129682 (2021).

26.An alternative approach of the e-nose training phase in odour impact assessment.. Chemical Engineering Transactions 30, (2012).

27.Muñoz, R. et al.. Monitoring techniques for odour abatement assessment. Water Research 44, 5129-5149 (2010).

28. Odour emissions characterization from wastewater treatment plants by different measurement methods. Chemical Engineering Transactions 40, (2014).

29.Zarra, T., Giuliani, S., Naddeo, V. \& Belgiorno, V. Control of odour emission in wastewater treatment plants by direct and undirected measurement of odour emission capacity. Water Science and Technology 66, 1627-1633 (2012).

30.Persaud, K. \& Dodd, G. Analysis of discrimination mechanisms in the mammalian olfactory 
system using a model nose. Nature 299, 352-355 (1982).

31.Measurement of odour emission capacity in wastewater treatment plants by multisensor array system. Environmental Engineering and Management Journal 12, (2013).

32.Zarra, T., Galang, M. G., Ballesteros, F., Belgiorno, V. \& Naddeo, V. Environmental odour management by artificial neural network - A review. Environment International 133, 105189 (2019).

33.Gardner, J. W. \& Bartlett, P. N. A brief history of electronic noses. Sensors and Actuators B: Chemical 18, 210-211 (1994).

34.Röck, F., Barsan, N. \& Weimar, U. Electronic Nose: Current Status and Future Trends. Chemical Reviews 108, 705-725 (2008).

35.luid dynamic simulation of odour measurement chamber. Chemical Engineering Transactions 40, (2014).

36.Baietto, M. \& Wilson, A. Electronic-Nose Applications for Fruit Identification Ripeness and Quality Grading. Sensors 15, 899-931 (2015).

37.Kiani, S., Minaei, S. \& Ghasemi-Varnamkhasti, M. Application of electronic nose systems for assessing quality of medicinal and aromatic plant products: A review. Journal of Applied Research on Medicinal and Aromatic Plants 3, 1-9 (2016).

38.Montuschi, P., Mores, N., Trové, A., Mondino, C. \& Barnes, P. J. The Electronic Nose in Respiratory Medicine. Respiration 85, 72-84 (2013).

39.Jia, P. et al.. Feature extraction of wound infection data for electronic nose based on a novel weighted KPCA. Sensors and Actuators B: Chemical 201, 555-566 (2014).

40.Deshmukh, S., Bandyopadhyay, R., Bhattacharyya, N., Pandey, R. A. \& Jana, A. Application of electronic nose for industrial odors and gaseous emissions measurement and monitoring - An 
overview. Talanta 144, 329-340 (2015).

41.Licen, S. et al.. Odor control map: Self organizing map built from electronic nose signals and integrated by different instrumental and sensorial data to obtain an assessment tool for real environmental scenarios. Sensors and Actuators B: Chemical 263, 476-485 (2018).

42.Gobbi, E. et al.. Rapid diagnosis of Enterobacteriaceae in vegetable soups by a metal oxide sensor based electronic nose. Sensors and Actuators B: Chemical 207, 1104-1113 (2015).

43.Loutfi, A., Coradeschi, S., Mani, G. K., Shankar, P. \& Rayappan, J. B. B. Electronic noses for food quality: A review. Journal of Food Engineering 144, 103-111 (2015).

44.Sokolova, M. \& Lapalme, G. A systematic analysis of performance measures for classification tasks. Information Processing \& Management 45, 427-437 (2009).

45.Majchrzak, T., Wojnowski, W., Dymerski, T., Gebicki, J. \& Namieśnik, J. Electronic noses in classification and quality control of edible oils: A review. Food Chemistry 246, 192-201 (2018). 
Figure Captions

Figure 1. Oil refinery plant 
Figures

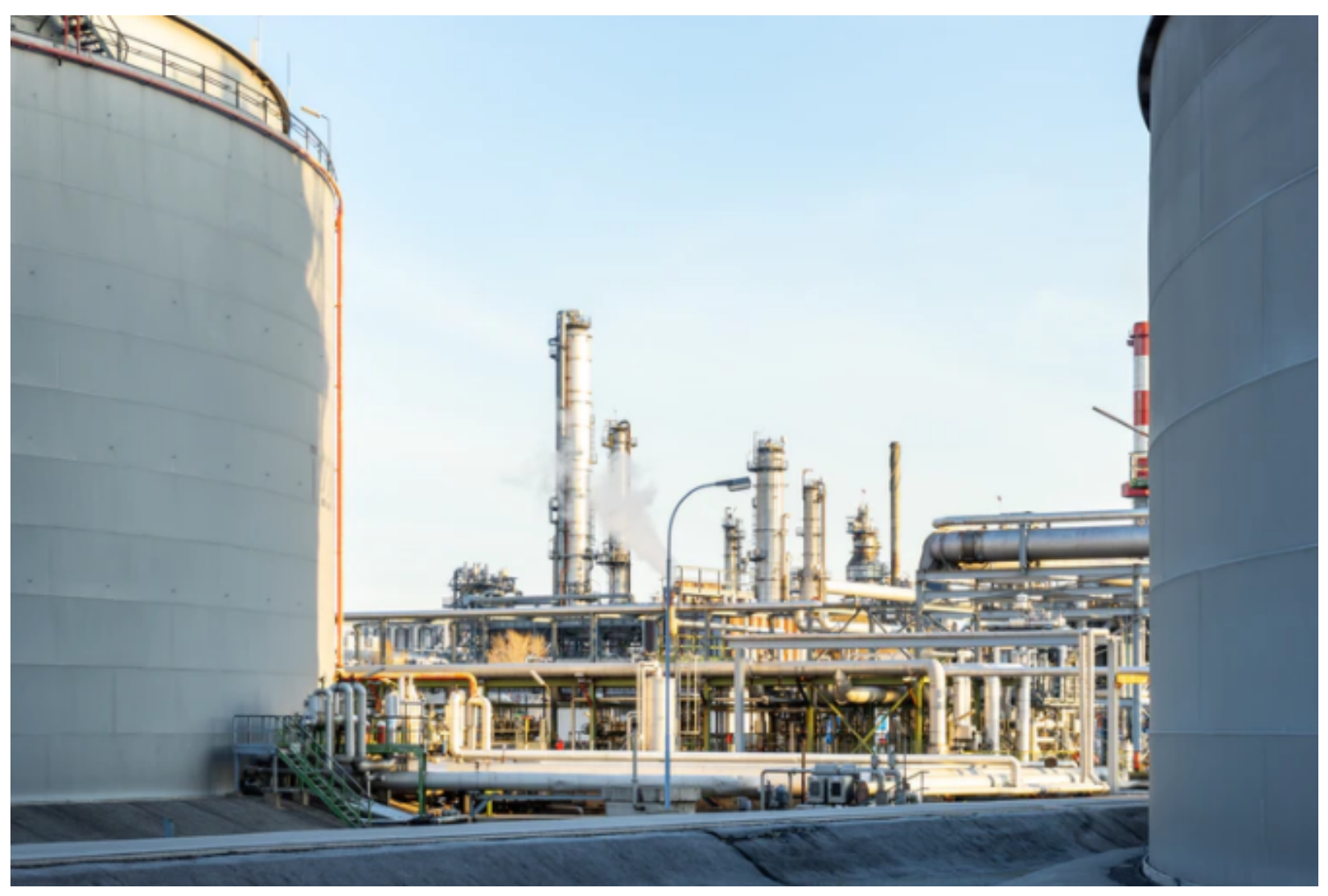

Figure 1: Oil refinery plant 